\title{
A MAXIMAL FUNCTION CHARACTERIZATION OF THE CLASS $H^{p}\left({ }^{1}\right)$
}

\author{
BY \\ D. L. BURKHOLDER, R. F. GUNDY AND M. L. SILVERSTEIN
}

\begin{abstract}
Let $u$ be harmonic in the upper half-plane and $0<p<\infty$. Then $u=\operatorname{Re} F$ for some analytic function $F$ of the Hardy class $H^{p}$ if and only if the nontangential maximal function of $u$ is in $L^{p}$. A general integral inequality between the nontangential maximal function of $u$ and that of its conjugate function is established.
\end{abstract}

Hardy and Littlewood have shown [6] ([12, I, p. 278]) that if $F(z)$ is an analytic function in the unit disc $|z|<1$, and $\Omega_{\sigma}(\theta)$ is the Stolz domain given by the interior of the smallest convex set containing the disc $|z|<\sigma$ and the point $e^{i \theta}$, then

$$
\int_{0}^{2 \pi} \sup _{z \in \Omega_{\sigma}(\theta)}|\operatorname{Re} F(z)|^{p} d \theta \leqq C_{\sigma, p} \sup _{0<r<1} \int_{0}^{2 \pi}\left|F\left(r e^{i \theta}\right)\right|^{p} d \theta
$$

for all $p>0,0<\sigma<1$. In this paper we prove the converse inequality which, together with the above theorem of Hardy and Littlewood, gives a maximal function characterization of the Hardy class $H^{p}$. We also prove the analogous inequality for the upper half-plane. Since the latter is the more difficult case, we treat it first.

In order to state our principal result we require some definitions. Recall that an analytic function $F(z)$ belongs to the class $H^{p}, p>0$, in the upper half-plane $\operatorname{Im} z>0$ if it is defined there and

$$
\sup _{y>0} \int_{-\infty}^{\infty}|F(x+i y)|^{p} d x<\infty .
$$

If $f(z)$ is any function defined in the upper half-plane, its nontangential maximal function is given by

$$
N_{\alpha}(f)(x)=\sup _{z \in \Gamma_{\alpha}(x)}|f(z)|
$$

where $\Gamma_{\alpha}(x)$ is the cone $\{z=s+i y:|s-x|<\alpha y\}$.

The function $N_{\alpha}(f)$ belongs to $L^{p}, p>0$, if

$$
\int_{-\infty}^{\infty}\left|N_{\alpha}(f)\right|^{p} d x<\infty
$$

Received by the editors September 1, 1970.

AMS 1969 subject classifications. Primary 3067, 3110, 6062.

Key words and phrases. Hardy class, harmonic function, conjugate harmonic function, nontangential maximal function, Brownian motion, martingale.

${ }^{(1)}$ This research was supported in part by the National Science Foundation under grants GP 23835, GP 19222, and GP 14022.

Copyright (C) 1971, American Mathematical Society 
THEOREM 1. Let $u$ be harmonic in the upper half-plane and $0<p<\infty$. Then $u=\operatorname{Re} F$ for some analytic function $F$ of the class $H^{p}$ if and only if $N_{\alpha}(u)$ belongs to $L^{p}$ for some $\alpha>0$. More specifically,

(a) if $u=\operatorname{Re} F$, then

$$
c_{\alpha, p} \int_{-\infty}^{\infty}\left|N_{\alpha}(u)\right|^{p} d x \leqq \sup _{y>0} \int_{-\infty}^{\infty}|F(x+i y)|^{p} d x
$$

for all $\alpha>0$;

(b) if for some $\alpha>0, N_{\alpha}(u)$ belongs to $L^{p}$, then there exists a conjugate harmonic function $\tilde{u}$ such that $F=u+i \tilde{u}$ is analytic in the upper half-plane and

$$
\sup _{y>0} \int_{-\infty}^{\infty}|F(x+i y)|^{p} d x \leqq C_{\alpha, p} \int_{-\infty}^{\infty}\left|N_{\alpha}(u)\right|^{p} d x .
$$

Part (a) is known and is the half-plane analogue of the theorem of Hardy and Littlewood. For the case $p>1$, part (b) follows from standard facts about the Hilbert transform. Therefore, the novelty of our result is the case $0<p \leqq 1$, which seems to require different methods. Our approach uses some ideas from probability theory. We obtain (a) and (b) in a unified way, as well as the following result.

THEOREM 2. Let $\Phi$ be a nondecreasing function such that

$$
\Phi(b)=\int_{0}^{b} \varphi(\lambda) d \lambda, \quad 0 \leqq b \leqq \infty,
$$

$0<\Phi(1)<\infty$, for some nonnegative, measurable function $\varphi$ satisfying the growth condition $\varphi(2 \lambda) \leqq c \varphi(\lambda)$ for all $\lambda>0$. If $u$ is harmonic in the upper half-plane, then there exists a conjugate function $\tilde{u}$ such that $F=u+i \tilde{u}$ is analytic in the upper halfplane and, for every $\alpha>0$,

$$
\int_{-\infty}^{\infty} \Phi\left(N_{\alpha}(\tilde{u})\right) d x \leqq C \int_{-\infty}^{\infty} \Phi\left(N_{\alpha}(u)\right) d x .
$$

The constant $C$ depends only on $\alpha$ and the growth constant for $\varphi$.

Note that the class of functions $\Phi$ described in Theorem 2 includes the powers $\Phi(b)=b^{p}, p>0$, as special cases.

A preliminary result that is of interest in its own right is given in $§ 1$. Stated as Theorem 3, it asserts that the distribution function of the nontangential maximal function is equivalent to the distribution function of another maximal function involving Brownian motion.

$\S 2$ contains the proof of Theorem 1; an alternative approach to the probabilistic part of the argument is presented in $\$ 3$. $\$ 4$ contains the essentials of a proof of Theorem 2. Since the argument follows the pattern of the proof of Theorem 1, we give only an outline. $\$ 5$ contains statements for the unit disc corresponding to Theorems 1, 2, and 3. Some remarks are made; for example, we show that while Theorem 2 holds in the unit disc for the nontangential maximal function, it does 
not hold for the radial maximal function. It would be interesting to establish the corresponding result for Theorem 1.

1. Preliminaries. For any point $z=x+i y$ with $y>0$, let $\left\{z_{t}, t \geqq 0\right\}$ be complexvalued Brownian motion started at $z$ and stopped the first time it hits the real axis $y=0$. The transition density for this process in the open upper half-plane is easily computed using the reflection principle and is given by the formula [5]

$$
q(t, \xi, \eta)=(2 \pi t)^{-1}\left[\exp \left(-|\eta-\xi|^{2} / 2 t\right)-\exp \left(-|\bar{\eta}-\xi|^{2} / 2 t\right)\right]
$$

where $\bar{\eta}$ is the complex conjugate of $\eta$. We let $P_{x+i y}$ denote the Wiener measure induced by the family $q(t, \xi, \eta), t>0$, and $\delta_{x+i y}$, the unit mass at $z=x+i y$. That is, $P_{x+i y}$ is a measure on the space of paths of the process $\left\{z_{t}, t \geqq 0\right\}$.

If $u$ is harmonic in the upper half-plane, define the Brownian maximal function and gradient function for $u$ as follows:

$$
\begin{gathered}
u^{*}=u_{x+i y}^{*}=\sup _{0 \leqq t<\tau_{0}}\left|u\left(z_{t}\right)\right|, \\
S(u)=S_{x+i y}(u)=\left[u^{2}(x+i y)+\int_{0}^{\tau_{0}}|\operatorname{grad} u|^{2}\left(z_{t}\right) d t\right]^{1 / 2},
\end{gathered}
$$

where $\tau_{0}$ is the stopping time $\tau_{0}=\inf \left\{t: \operatorname{Im} z_{t}=0\right\}$. For any function $v$ of the process $\left\{z_{t}, t \geqq 0\right\}$, we write

$$
\|v\|_{p}=\left(\int|v|^{p} d P_{x+i y}\right)^{1 / p}, \quad 0<p<\infty .
$$

Finally, $m($ ) always denotes Lebesgue measure on the real axis or on lines parallel to it.

The following result is used in the proofs of Theorems 1 and 2, but is of independent interest.

THEOREM 3. Let $u$ be harmonic in the upper half-plane. Then, for $\alpha>0$,

$$
c_{\alpha} m\left(N_{\alpha}(u)>\lambda\right) \leqq \sup _{y>0} \int_{-\infty}^{\infty} P_{x+i y}\left(u^{*}>\lambda\right) d x \leqq C_{\alpha} m\left(N_{\alpha}(u)>\lambda\right) .
$$

The expression $\sup _{y>0} \int_{-\infty}^{\infty} P_{x+i y}\left(u^{*}>\lambda\right) d x$ can be interpreted as the distribution function for the Brownian maximal function corresponding to Brownian motion "starting at infinity" in the sense of Hunt [8, p. 334]. The proof of Theorem 3 rests on two lemmas.

LEMMA 1. Let $z_{\infty}$ denote the terminal position of the process $\left\{z_{t}, t \geqq 0\right\}$ on the line $y=0$. The random variable $z_{\infty}$ has a distribution with density given by the Poisson kernel

$$
p_{x+i y}(s)=\frac{1}{\pi} \frac{y}{\left(|x-s|^{2}+y^{2}\right)}
$$


and for any Lebesgue integrable function $f$ we have the formula

$$
\int_{-\infty}^{\infty} f(s) d s=\int_{-\infty}^{\infty} \int f\left(z_{\infty}\right) d P_{x+i y} d x
$$

This lemma is well known and follows from a general theorem due to Kakutani that identifies harmonic measure with the hitting distribution of Brownian motion. See [3] for details.

Lemma 2. Let u be harmonic in the upper half-plane.

(a) If

$$
a(\lambda)=m\left(N_{\alpha}(u)>\lambda\right)
$$

is finite for some $\alpha>0$ and $\lambda>0$, then $|u(x+i y)| \leqq \lambda$ for $y>(2 \alpha)^{-1} a(\lambda)$.

(b) If

$$
b(\lambda)=\sup _{y>0} \int_{-\infty}^{\infty} P_{x+i y}\left(u^{*}>\lambda\right) d x
$$

is finite for some $\lambda>0$, then $|u(x+i y)| \leqq \lambda$ for $y>C b(\lambda)$.

Proof. Let $A=\left\{x: N_{\alpha}(u)(x)>\lambda\right\}, A^{\prime}$ its complement, and $B=\bigcup_{x \in A^{\prime}} \Gamma_{\alpha}(x)$. The boundary of $B$ is saw-toothed, and consists of $A^{\prime}$ and the upper sides of a countable number of isosceles triangles. An elementary calculation shows that the heights of these triangles are all bounded by $y=(2 \alpha)^{-1} a(\lambda)$, and that $|u| \leqq \lambda$ above this line.

To prove (b), we make the following preliminary observations: Let $Q$ be the square with vertices $( \pm 1,0),( \pm 1,2)$, and $\gamma$ a Jordan arc with one endpoint at the origin, the other off the real axis on the boundary of $Q$, and all other points of $\gamma$ in the interior of $Q$. The square is thereby divided into two regions, $Q_{R}$ and $Q_{L}$ with the segment $R=\{x: 0 \leqq x \leqq 1\}$ forming part of the boundary of $Q_{R}$ and $L=\{x:-1 \leqq x \leqq 0\}$ part of the boundary of $Q_{L}$. Therefore, we have

$$
P_{z}\left(z_{t} \text { hits } \gamma\right) \geqq \min \left(P_{z}\left(z_{t} \text { leaves } Q \text { along } R\right), P_{z}\left(z_{t} \text { leaves } Q \text { along } L\right)\right)
$$

for $z$ belonging to $Q$ and the line $\operatorname{Im} z=1$. Since the right-hand side of this inequality is greater than some positive constant $d$ for all $z$ on the line segment $J=\left\{z:-\frac{1}{2} \leqq \operatorname{Re} z \leqq \frac{1}{2}, \operatorname{Im} z=1\right\}$, it follows that

$$
\inf _{z \in J} P_{z}\left(z_{t} \text { hits } \gamma\right) \geqq d
$$

and

$$
\inf _{z \in J} P_{z}\left(z_{t} \text { leaves } Q \text { along the bottom edge }\right) \geqq d \text {. }
$$

We now show that (b) holds with $C=5 d^{-1}$. We first assume that the open set $D=\{z:|u(z)|>\lambda\}$ is connected and that $b(\lambda)>0$. Let $h_{I}$ be the length of the projection of $D$ on the imaginary axis, and $h_{R}$ the length of the projection of $D$ on the 
real axis. There are two possibilities: Either $h_{I}>4 d^{-1} b(\lambda)$ or $h_{I} \leqq 4 d^{-1} b(\lambda)$. Suppose that the first possibility holds. Then there exists a Jordan arc $\gamma$ with one endpoint $z_{0}$ on a line $y=l$ and the other endpoint on the line $y=l+4 d^{-1} b(\lambda)$ such that all interior points of $\gamma$ lie in $D$ and strictly above the line $y=l$. Dilate the square $Q$ by a factor of $2 d^{-1} b(\lambda)$ and translate it, bringing the origin to the point $z_{0}$. The arc $\gamma$ then divides the new square and the homogeneity properties of Brownian motion, together with (1.2), imply

$$
P_{x+i\left(l+2 d^{-1} b(\lambda)\right)}\left(u^{*}>\lambda\right) \geqq P_{x+i\left(l+2 d^{-1} b(\lambda)\right)}\left(z_{t} \text { hits } \gamma\right) \geqq d
$$

for $x$ in the segment symmetric about $z_{0}$ of length $2 d^{-1} b(\lambda)$. Therefore,

$$
\begin{aligned}
b(\lambda) & \geqq \int_{x_{0}-d^{-1 b(\lambda)}}^{x_{0}+d^{-1} b(\lambda)} P_{x+i\left(l+2 d^{-1} b(\lambda)\right)}\left(z_{t} \text { hits } \gamma\right) d x \\
& \geqq 2 d^{-1} b(\lambda) d=2 b(\lambda) .
\end{aligned}
$$

This contradiction implies $h_{I} \leqq 4 d^{-1} b(\lambda)$. Therefore, we may assume that $h_{I}$ $\leqq 4 d^{-1} b(\lambda)$ and suppose that (b) does not hold with $C=5 d^{-1}$. It follows by the maximum principle that $h_{R}=\infty$, since otherwise the set $D$ would be bounded and its closure strictly contained in the interior of the upper half-plane. Therefore, there exists a Jordan arc $\gamma$ in $D$ that extends to infinity either to the right or left, and whose oscillation in the vertical direction is no greater than $4 d^{-1} b(\lambda)$. Dilate the square $Q$ by $8 d^{-1} b(\lambda)$. Because $h_{R}=\infty$ and $h_{I} \leqq 4 d^{-1} b(\lambda)$, it is possible to translate the dilated square to have base along $y=l$ so that the arc $\gamma$ divides it along the vertical edges into two connected regions, $U$ and $L$, such that the upper region $U$ contains the horizontal midline of the square. The homogeneity properties of Brownian motion, together with (1.3), imply

$$
\begin{aligned}
b(\lambda) & \geqq \int_{x_{0}-4 d^{-1} b(\lambda)}^{x_{0}+4 d^{-1} b(\lambda)} P_{x+i\left(l+8 d^{-1} b(\lambda)\right)}\left(u^{*}>\lambda\right) d x \\
& \geqq \int_{x_{0}-4 d^{-1} b(\lambda)}^{x_{0}+4 d^{-1} b(\lambda)} P_{x+i\left(l+8 d^{-1} b(\lambda)\right)}\left(z_{t} \text { hits } \gamma\right) d x \\
& \geqq 8 d^{-1} b(\lambda) d=8 b(\lambda)
\end{aligned}
$$

where $z=x_{0}+i\left(l+8 d^{-1} b(\lambda)\right)$ is the midpoint of the dilated square. This contradiction implies that (b) holds if $D$ is connected, and the general case follows by applying the above argument to each component.

Proof of Theorem 3. We fix $\lambda>0$ throughout the proof. To prove the right-hand inequality, assume that $m\left(N_{\alpha}(u)>\lambda\right)=a(\lambda)<\infty$. The set $D=\{z:|u(z)|>\lambda\}$ is contained in the complement of $B$, defined in the proof of part (a) of Lemma 2. If $y>(2 \alpha)^{-1} a(\lambda)$, then

$$
\begin{aligned}
P_{x+i y}\left(u^{*}>\lambda\right) & =P_{x+i y}\left(z_{t} \text { enters } D \text { for some } t>0\right) \\
& \leqq P_{x+i y}\left(z_{t} \in \partial B \text { for some } t>0\right)
\end{aligned}
$$


where $\partial B$ indicates the saw-toothed part of the boundary of $B$. Also note that

$$
\inf _{z \in \partial B} P_{z}\left(z_{\infty} \in A\right) \geqq c_{\alpha}>0,
$$

a fact that follows from the homogeneity properties of the Poisson kernel and Lemma 1.

Consider the stopping time $\tau=\inf \left\{t: z_{t} \in \partial B\right\}$. We use Lemma 1, the strong Markov property of Brownian motion [7], (1.4), and (1.5) in the following computation. If $y>(2 \alpha)^{-1} a(\lambda)$, then

$$
\begin{aligned}
m\left(N_{\alpha}(u)>\lambda\right) & =\int_{-\infty}^{\infty} P_{x+i y}\left(z_{\infty} \in A\right) d x \\
& =\int_{-\infty}^{\infty} P_{x+i y}\left(z_{\infty} \in A, \tau<\infty\right) d x \\
& =\int_{-\infty}^{\infty} \int_{\partial B} P_{x+i y}\left(z_{\tau} \in d z\right) P_{z}\left(z_{\infty} \in A\right) d x \\
& \geqq c_{\alpha} \int_{-\infty}^{\infty} P_{x+i y}\left(u^{*}>\lambda\right) d x .
\end{aligned}
$$

Finally, note that the last expression is nondecreasing in $y$ : If $0<r<y$, then, by another use of the strong Markov property, we have that

$$
P_{x+i y}\left(u^{*}>\lambda\right) \geqq \int_{-\infty}^{\infty} P_{s+i r}\left(u^{*}>\lambda\right) p_{x+i(y-r)}(s) d s
$$

Integrating both sides with respect to $x$, we obtain

$$
\int_{-\infty}^{\infty} P_{x+i y}\left(u^{*}>\lambda\right) d x \geqq \int_{-\infty}^{\infty} P_{s+i r}\left(u^{*}>\lambda\right) d s, \quad 0<r<y .
$$

This completes the proof of the right-hand side of Theorem 3.

For the left-hand side, we introduce a conditional Brownian motion process with transition density

$$
q^{s}(t, \xi, \eta)=q(t, \xi, \eta) p_{\eta}(s) / p_{\xi}(s)
$$

where $q(t, \xi, \eta)$ is the density (1.1) and $p_{\eta}(s)$ is the Poisson kernel. Conditional processes of this type have been discussed by Doob [4], [5]. The function $q^{s}$ is the transition density for Brownian motion conditioned to hit the real axis at $s,-\infty<s$ $<\infty$. The conditioned process has the strong Markov property [4, p. 436] and the obvious homogeneity properties under real translations and dilations. We let $P_{x+i y}^{s}$ denote the Wiener measure associated with the density $q^{s}$.

The following observation may be verified using the strong Markov property of Brownian motion. Let $k$ be the segment formed by the intersection of the line $\operatorname{Im} z=1$ with the cone $\Gamma_{\alpha}(0)$. Then

$$
P_{x+i y}^{0}\left(z_{t} \text { does not hit } k\right) \geqq C_{\alpha}>0
$$


for $y \geqq 2$. This inequality, together with the homogeneity properties of the density $q^{s}$, implies that if $K$ is the segment formed by the intersection of the line $\operatorname{Im} z=r$ with the cone $\Gamma_{\alpha}(s)$, then, for $y \geqq 2 r$,

$$
P_{x+i y}^{s}\left(z_{t} \text { does not hit } K\right) \geqq C_{\alpha}>0
$$

where the constant $C_{\alpha}$ is the same as in (1.7).

In order to prove the left-hand inequality, we may assume that

$$
\sup _{y>0} \int_{-\infty}^{\infty} P_{x+i y}\left(u^{*}>\lambda\right) d x<\infty .
$$

Let $s \in A$, that is, assume $N_{\alpha}(u)(s)>\lambda$. Denote by $l_{s}$ a horizontal segment across $\Gamma_{\alpha}(s)$ such that $|u(z)|>\lambda$ for some $z$ on $l_{s}$. By Lemma 2 and (1.8),

$$
P_{x+i y}^{s}\left(z_{t} \text { does not hit } l_{s}\right) \geqq C_{\alpha}>0
$$

for $y>2 C b(\lambda)$, where $C_{\alpha}=C_{(1.8)}$ and $C$ is the constant specified in Lemma 2(b).

We complete the proof of the left-hand inequality by using the maximum principle and the fact that enough Brownian paths to $s$ and their reflections avoid the segment $l_{s}$. If $z_{t}=x_{t}+i y_{t}, t \geqq 0$, define the reflected process $\hat{z}_{t}=2 s-x_{t}+i y_{t}$. It is easily verified that

$$
P_{x+i y}^{s}(\hat{z} \in E)=P_{2 s-x+i y}^{s}\left(z_{t} \in E\right) ;
$$

in fact, the conditional distribution of $\left\{\hat{z}_{t}, t \geqq 0\right\}$ is the same as that of $\left\{z_{t}, t \geqq 0\right\}$ with initial point reflected about $s$. If the process $\left\{z_{t}, t \geqq 0\right\}$ is started on the line $\operatorname{Im} z=2 C b(\lambda)$ and terminates at $s$ without hitting $l_{s}$, then the same is true for $\hat{z}_{t}$ since $l_{s}$ is symmetric about $s$. In this case, the union of the paths of $z_{t}, \hat{z}_{t}$, and the line $\operatorname{Im} z=2 C b(\lambda)$, contains a closed curve around the segment $l_{s}$. Since $|u(z)| \leqq \lambda$ on $\operatorname{Im} z=2 C b(\lambda)$ and $|u(z)|>\lambda$ at an endpoint of $l_{s}$, the maximum principle implies that, for these paths,

$$
*^{*} u \vee u^{*}=\left(\sup _{0<t<\tau_{0}}|u(\hat{z})|\right) \vee\left(\sup _{0<t<\tau_{0}}\left|u\left(z_{t}\right)\right|\right)>\lambda .
$$

Therefore, if $y>2 C b(\lambda)$,

$$
\begin{aligned}
P_{2 s-x+i y}^{s}\left(u^{*}>\lambda\right)+P_{x+i y}^{s}\left(u^{*}>\lambda\right) & =P_{x+i y}^{s}\left({ }^{*} u>\lambda\right)+P_{x+i y}^{s}\left(u^{*}>\lambda\right) \\
& \geqq P_{x+i y}^{s}\left({ }^{*} u \vee u^{*}>\lambda\right) \\
& \geqq P_{x+y y}^{s}\left(z_{t} \text { does not hit } l_{s}\right) \geqq C_{\alpha}>0
\end{aligned}
$$

by (1.9). Let $E_{x+i y}$ denote expectation relative to $P_{x+i y}$, let $P_{x+i y}\left(\cdot \mid z_{\infty}\right)$ denote the conditional probability relative to the random variable $z_{\infty}$, and let $I_{A}(s)$ be the indicator function of the set $A=\left\{x: N_{\alpha}(u)>\lambda\right\}$. Then by Lemma 1, 


$$
\begin{aligned}
2 \int_{-\infty}^{\infty} P_{x+i y}\left(u^{*}>\lambda\right) d x & =2 \int_{-\infty}^{\infty} E_{x+i y}\left[P_{x+i y}\left(u^{*}>\lambda \mid z_{\infty}\right)\right] d x \\
& \geqq 2 \int_{-\infty}^{\infty} E_{x+i y}\left[I_{A}\left(z_{\infty}\right) P_{x+i y}\left(u^{*}>\lambda \mid z_{\infty}\right)\right] d x \\
& =\frac{2}{\pi} \int_{-\infty}^{\infty} \int_{-\infty}^{\infty} I_{A}(s) P_{x+i y}^{s}\left(u^{*}>\lambda\right) \frac{y}{y^{2}+(x-s)^{2}} d s d x \\
& =\frac{1}{\pi} \int_{-\infty}^{\infty} I_{A}(s)\left[\int_{-\infty}^{\infty} P_{2 s-x+i y}^{s}\left(u^{*}>\lambda\right) \frac{y}{y^{2}+(x-s)^{2}} d x\right. \\
& \geqq \int_{-\infty}^{\infty} I_{A}(s) C_{\alpha} \int_{-\infty}^{\infty} \frac{1}{\pi} \frac{y}{y^{2}+(x-s)^{2}} d x d s \\
& =C_{\alpha} m\left(N_{\alpha}(u)>\lambda\right),
\end{aligned}
$$

which implies the left-hand inequality of Theorem 3.

LEMMA 3. Suppose that $u$ is harmonic in the upper half-plane, and

$$
\int_{-\infty}^{\infty}\left|N_{\alpha}(u)\right|^{p} d x<\infty
$$

for some $\alpha>0$ and $p>0$. Then there exists a conjugate harmonic function $\tilde{u}$ such that $F=u+i \tilde{u}$ is analytic in the upper half-plane, and

$$
\lim _{y \rightarrow \infty} \tilde{u}(x+i y)=0
$$

uniformly in $x$.

The proof of Lemma 3 uses standard facts about harmonic functions in the upper half-plane. The hypothesis (1.10) and Lemma 2 imply that $u$ is bounded along every line $\operatorname{Im} z=\varepsilon>0$.

First, consider the case $p \leqq 1$; then $u$ is also in $L^{2}$ along every line $\operatorname{Im} z=\varepsilon$. Therefore $u$ has a conjugate function $\tilde{u}_{\varepsilon}$ in the half-plane $\operatorname{Im} z \geqq \varepsilon$ formed by taking the Poisson integral of the Hilbert transform of $u$ along the line $\operatorname{Im} z=\varepsilon$. Since the Hilbert transform of $u$ along $\operatorname{Im} z=\varepsilon$ is also in $L^{2}$, its Poisson integral $\tilde{u}_{\varepsilon}$ tends to zero uniformly in $x$ as $y$ tends to infinity; this fact may be verified directly from the Poisson integral formula. Therefore,

$$
F_{\varepsilon}(x+i y)=u(x+i y)+i \tilde{u}_{\varepsilon}(x+i y)
$$

is analytic in $\operatorname{Im} z>\varepsilon$. Finally, if $0<\varepsilon^{\prime}<\varepsilon$, then $F_{\varepsilon^{\prime}}=F_{\varepsilon}$ on the common domain since each has the same real part and both tend to zero as $y$ tends to infinity. Therefore, the functions $F_{\varepsilon}$ may be continued to a function $F$ that is analytic in the half-plane and satisfies (1.11).

If $p>1$, the same argument works with $\varepsilon=0$ and with $L^{2}$ replaced by $L^{p}$. 
LEMMA 4. Let $u$ be harmonic in the upper half-plane. For any initial point $z_{0}$, we have

$$
c_{p}\left\|S_{z_{0}}(u)\right\|_{p} \leqq\left\|u_{z_{0}}^{*}\right\|_{p} \leqq C_{p}\left\|S_{z_{0}}(u)\right\|_{p}
$$

for $0<p<\infty$.

Lemma 4 is a consequence of some martingale maximal inequalities from [2], and some facts about stochastic integrals. However, the use of stochastic integrals may be avoided by another approach, given in $\$ 3$, that uses the methods of [2] to prove directly the following consequence of Lemma 4 : Let $u$ and $\tilde{u}$ be conjugate harmonic functions in the upper half-plane. Then

$$
\left\|\sup _{0<t<\tau_{0}}\left|\tilde{u}\left(z_{t}\right)-\tilde{u}\left(z_{0}\right)\right|\right\|_{p} \leqq C_{p}\left\|_{0<t<\tau_{0}}\left|u\left(z_{t}\right)-u\left(z_{0}\right)\right|\right\|_{p}
$$

for $0<p<\infty$.

Proof of Lemma 4. It follows from Itô's formula for stochastic differentials that the process $\left\{u\left(z_{t}\right), t \geqq 0\right\}$ is a stochastic integral of the form

$$
u\left(z_{t}\right)=u\left(z_{0}\right)+\int_{0}^{t} \operatorname{grad} u\left(z_{t}\right) \cdot\left(d x_{t}, d y_{t}\right)
$$

where $z_{t}=x_{t}+i y_{t}$. (For a discussion of stochastic integrals and Itô's formula, see McKean [9].) To obtain Lemma 4 in the range $p>1$, we may use results of Millar [10, Theorem 7.1] and Doob's maximal inequalities for martingales. The double inequality may be extended to the range $0<p \leqq 1$ by using the extrapolation method of [2]. We give no more details here since an alternative approach is presented in $\$ 3$.

Inequality (1.12) follows directly from Lemma 4: Since $|\operatorname{grad} u|^{2}=|\operatorname{grad} \tilde{u}|^{2}$ implies $S_{z_{0}}\left(u-u\left(z_{0}\right)\right)=S_{z_{0}}\left(\tilde{u}-\tilde{u}\left(z_{0}\right)\right)$, we have

$$
\begin{aligned}
\left\|\sup _{0<t<\tau_{0}} \mid \tilde{u}\left(z_{t}\right)-\tilde{u}\left(z_{0}\right)\right\|_{p} & \leqq C_{p}\left\|S_{z_{0}}\left(\tilde{u}-\tilde{u}\left(z_{0}\right)\right)\right\|_{p} \\
& =C_{p}\left\|S_{z_{0}}\left(u-u\left(z_{0}\right)\right)\right\|_{p} \leqq C_{p}\left\|_{0<t<\tau_{0}} \mid u\left(z_{t}\right)-u\left(z_{0}\right)\right\|_{p} .
\end{aligned}
$$

2. Proof of Theorem 1. We prove part (b) first. Suppose that the right-hand side of (0.2) is finite. Theorem 3 implies

$$
\begin{aligned}
\sup _{y>0} \int_{-\infty}^{\infty}\left\|u_{x+i y}^{*}\right\|_{p}^{p} d x & =\sup _{y>0} \int_{-\infty}^{\infty} \int_{0}^{\infty} p \lambda^{p-1} P_{x+i y}\left(u^{*}>\lambda\right) d \lambda d x \\
& \leqq p \int_{0}^{\infty} \lambda^{p-1} \sup _{y>0} \int_{-\infty}^{\infty} P_{x+i y}\left(u^{*}>\lambda\right) d x d \lambda \\
& \leqq C_{\alpha} p \int_{0}^{\infty} \lambda^{p-1} m\left(N_{\alpha}(u)>\lambda\right) d \lambda \\
& =C_{\alpha} \int_{-\infty}^{\infty}\left|N_{\alpha}(u)\right|^{p} d x<\infty .
\end{aligned}
$$


We show that this, together with the information collected above, implies

$$
\sup _{y>0} \int_{-\infty}^{\infty}|\tilde{u}(x+i y)|^{p} d x \leqq C_{\alpha} \int_{-\infty}^{\infty}\left|N_{\alpha}(u)\right|^{p} d x .
$$

Fix $y>0$ and suppose that $0<y<r$; define

$$
\left|\tilde{u}_{r}(x+i y)\right|^{p}=\inf _{-\infty<s<\infty}|\tilde{u}(x+i y)-\tilde{u}(s+i r)|^{p} .
$$

By Lemma 3,

$$
\lim _{r \rightarrow \infty}\left|\tilde{u}_{r}(x+i y)\right|^{p}=|\tilde{u}(x+i y)|^{p},
$$

and by Fatou's lemma

$$
\int_{-\infty}^{\infty}|\tilde{u}(x+i y)|^{p} d x \leqq \liminf _{r \rightarrow \infty} \int_{-\infty}^{\infty}|\tilde{u},(x+i y)|^{p} d x .
$$

Let $\left\{z_{t}^{y}, t \geqq 0\right\}$ be Brownian motion started at the point $s+i$ ir and stopped the first time it reaches the line $\operatorname{Im} z=y$. Lemma 1, inequality (1.6), and the translation properties of Brownian motion imply

$$
\begin{aligned}
\int_{-\infty}^{\infty}\left|\tilde{u}_{r}(x+i y)\right|^{p} d x & \leqq \int_{-\infty}^{\infty} E_{s+i r}\left(\left|\tilde{u}\left(z_{\infty}^{y}\right)-\tilde{u}(s+i r)\right|^{p}\right) d s \\
& \leqq \int_{-\infty}^{\infty} E_{s+i r}\left(\sup _{t>0}\left|\tilde{u}\left(z_{t}^{y}\right)-\tilde{u}(s+i r)\right|^{p}\right) d s \\
& \leqq C_{p} \int_{-\infty}^{\infty} E_{s+i r}\left(\sup _{t>0}\left|u\left(z_{t}^{y}\right)-u(s+i r)\right|^{p}\right) d s \\
& \leqq 2^{p} C_{p} \int_{-\infty}^{\infty}\left\|u_{s+i r}^{*}\right\|_{p}^{p} d s,
\end{aligned}
$$

and therefore, by (2.2),

$$
\sup _{y>0} \int_{-\infty}^{\infty}|\tilde{u}(x+i y)|^{p} d x \leqq 2^{p} C_{p} \sup _{y>0} \int_{-\infty}^{\infty}\left\|u_{x+i y}^{*}\right\|_{p}^{p} d x .
$$

Finally, by (2.1),

$$
\sup _{y>0} \int_{-\infty}^{\infty}|\tilde{u}(x+i y)|^{p} d x \leqq C_{\alpha, p} \int_{-\infty}^{\infty}\left|N_{\alpha}(u)\right|^{p} d x,
$$

so that

$$
\begin{aligned}
\sup _{y>0} \int_{-\infty}^{\infty}|F(x+i y)|^{p} d x & =\sup _{y>0} \int_{-\infty}^{\infty}|u(x+i y)+i \tilde{u}(x+i y)|^{p} d x \\
& \leqq C_{\alpha, p} \int_{-\infty}^{\infty}\left|N_{\alpha}(u)\right|^{p} d x,
\end{aligned}
$$

which completes the proof of part (b) of Theorem 1. 
Part (a) of Theorem 1, due to Hardy and Littlewood, may also be proved using martingales. Suppose that $F(z)$ belongs to $H^{p}$ for some $p>0$; then $|F(z)|^{p / 2}$ is subharmonic in the upper half-plane and is bounded along the line $\operatorname{Im} z=y>0$. The process $\left\{\left|F\left(z_{t}^{y}\right)\right|^{p / 2}, t \geqq 0\right\}$ is an $L^{2}$-bounded submartingale so that by Doob's maximal inequalities for submartingales and Lemma 1 , for $r>y$,

$$
\begin{aligned}
\int_{-\infty}^{\infty} \int \sup _{t>0}\left|u\left(z_{t}^{y}\right)\right|^{p} d P_{s+\imath r} d s & \leqq \int_{-\infty}^{\infty} \int_{t>0}\left|F\left(z_{t}^{y}\right)\right|^{p} d P_{s+i r} d s \\
& \leqq C_{p} \int_{-\infty}^{\infty} \int\left(\left|F\left(z_{\infty}^{y}\right)\right|^{p / 2}\right)^{2} d P_{s+i r} d s \\
& =C_{p} \int_{-\infty}^{\infty}|F(x+i y)|^{p} d x .
\end{aligned}
$$

Therefore

$$
\int_{-\infty}^{\infty}\left\|u_{s+i r}^{*}\right\|_{p}^{p} d s \leqq C_{p} \sup _{y>0} \int_{-\infty}^{\infty}|F(x+i y)|^{p} d x
$$

and Theorem 3, together with (1.6), implies

$$
\begin{aligned}
\int_{-\infty}^{\infty}\left|N_{\alpha}(u)\right|^{p} d x & \leqq C_{\alpha} \sup _{y>0} \int_{-\infty}^{\infty}\left\|u_{x+i y}^{*}\right\|_{p}^{p} d x \\
& \leqq C_{\alpha, p} \sup _{y>0} \int_{-\infty}^{\infty}|F(x+i y)|^{p} d x,
\end{aligned}
$$

which completes the proof of Theorem 1.

3. An alternative approach. Here we give an alternative proof of (1.12), one of the key inequalities leading to Theorem 1. The proof is self-contained and is a particularly simple application of the methods developed in [2].

THEOREM 4. Let $u$ be harmonic in some connected open set $D$ of the complex plane and $\tilde{u}$ a conjugate function in the sense that $F=u+i \tilde{u}$ is analytic in $D$. Let $\left\{Z_{t}, 0 \leqq t<\infty\right\}$ be Brownian motion in the plane starting from the point $x+i y$ in $D$ and $\tau$ a stopping time of this process such that if $t<\tau$, then $Z_{t} \in D$. Then, for $0<p<\infty$,

$$
\left\|\sup _{0 \leqq t<\tau}\left|\tilde{u}\left(Z_{t}\right)-\tilde{u}(x+i y)\left\|_{p} \leqq c_{p}\right\| \sup _{0 \leqq t<\tau}\right| u\left(Z_{i}\right)-u(x+i y)\right\|_{p} .
$$

The choice of $c_{p}$ depends only on $p$.

This implies (1.12).

Proof. In the proof we may assume that $F(x+i y)=0$ and, by the monotone convergence theorem and the topology of the plane, that there is an open set $D_{0}$ containing $x+i y$ whose closure is a compact subset of $D$ such that $Z_{t} \in D_{0}$ for $t<\tau$. Otherwise, we could replace $\tau$ by $\tau_{n}$ where $\tau_{n}$ has the desired property and $\tau_{n}$ increases to $\tau$ as $n$ increases.

Let $z_{\iota}=Z_{\tau \wedge t}, u^{*}=\sup _{0 \leqq t<\infty}\left|u\left(z_{t}\right)\right|$, and $F^{*}=\sup _{0 \leqq t<\infty}\left|F\left(z_{t}\right)\right|$. We show that $\left\|F^{*}\right\|_{p} \leqq c_{p}\left\|u^{*}\right\|_{p}$, which implies Theorem 4 . 
First, we note that if $\mu$ and $\nu$ are stopping times of the process $\left\{z_{t}, 0 \leqq t<\infty\right\}$ and $\mu \leqq \nu$, then

$$
\left\|\tilde{u}\left(z_{v}\right)-\tilde{u}\left(z_{\mu}\right)\right\|_{2}=\left\|u\left(z_{v}\right)-u\left(z_{\mu}\right)\right\|_{2} .
$$

To prove this, note that, since $F^{2}$ is analytic in $D$, the process $\left\{F^{2}\left(z_{t}\right), 0 \leqq t<\infty\right\}$ is a martingale (Doob [3, Theorem 4.3]). Since this martingale is uniformly bounded,

so that

$$
0=F^{2}(x+i y)=\lim _{t \rightarrow \infty} \int F^{2}\left(z_{\mu \wedge t}\right) d P=\int F^{2}\left(z_{\mu}\right) d P
$$

$$
\left\|u\left(z_{\mu}\right)\right\|_{2}=\left\|\tilde{u}\left(z_{\mu}\right)\right\|_{2},
$$

with a similar formula holding for $\nu$. Also, $\left\{u\left(z_{\mu}\right), u\left(z_{v}\right)\right\}$ is a martingale and from the orthogonality of its increments it follows that

$$
\int u\left(z_{\mu}\right) u\left(z_{v}\right) d P=\int u^{2}\left(z_{\mu}\right) d P .
$$

A similar formula holds for $\tilde{u}$ and (3.2) now follows by a simple computation.

We now come to the crucial step in the proof.

(i) Let $\alpha \geqq 1$ and $\beta>1$. Then

$$
P\left(F^{*}>\lambda\right) \leqq c P\left(c u^{*}>\lambda\right)
$$

for all $\lambda>0$ satisfying

$$
P\left(F^{*}>\lambda\right) \leqq \alpha P\left(F^{*}>\beta \lambda\right) .
$$

The choice of $c$ depends only on $\alpha$ and $\beta$.

The stopping times

$$
\begin{aligned}
& \mu=\inf \left\{t:\left|F\left(z_{t}\right)\right|>\lambda\right\}, \\
& \nu=\inf \left\{t:\left|F\left(z_{t}\right)\right|>\beta \lambda\right\}
\end{aligned}
$$

satisfy $\mu \leqq \nu ;\left|F\left(z_{\mu}\right)\right|=\lambda$ on the set $\{\mu<\infty\}=\left\{F^{*}>\lambda\right\}$, and $\left|F\left(z_{v}\right)\right|=\beta \lambda$ on $\left\{F^{*}>\beta \lambda\right\}$. Accordingly, by (3.2) and (3.4),

$$
\begin{aligned}
\int_{\left\{F^{*}>\lambda\right\}}\left[u\left(z_{v}\right)-u\left(z_{u}\right)\right]^{2} d P & =\left\|u\left(z_{v}\right)-u\left(z_{\mu}\right)\right\|_{2}^{2} \\
& =\frac{1}{2}\left\|F\left(z_{v}\right)-F\left(z_{\mu}\right)\right\|_{2}^{2} \geqq \frac{1}{2}(\beta \lambda-\lambda)^{2} P\left(F^{*}>\beta \lambda\right) \\
& \geqq c \lambda^{2} P\left(F^{*}>\lambda\right) .
\end{aligned}
$$

Also,

$$
\begin{aligned}
\int_{\left\{F^{*}>\lambda\right\}}\left[u\left(z_{v}\right)-u\left(z_{\mu}\right)\right]^{4} d P & \leqq\left\|F\left(z_{v}\right)-F\left(z_{\mu}\right)\right\|_{4}^{4} \\
& \leqq c \lambda^{4} P\left(F^{*}>\lambda\right) .
\end{aligned}
$$

Therefore, by a lemma of Paley and Zygmund [12, Chapter V, 8.26],

$$
P\left(F^{*}>\lambda\right) \leqq c P\left(c\left|u\left(z_{v}\right)-u\left(z_{\mu}\right)\right|>\lambda\right) .
$$

Since $\left|u\left(z_{v}\right)-u\left(z_{u}\right)\right| \leqq 2 u^{*}$, we obtain (3.3). 
(ii) We can now complete the proof of the theorem. Let $\alpha=2^{p+1}, \beta=2$, and

$$
B=\left\{\lambda>0: P\left(F^{*}>\lambda\right) \leqq \alpha P\left(F^{*}>\beta \lambda\right)\right\} .
$$

The following fact is elementary:

$$
\left\|F^{*}\right\|_{p}^{p}=\int_{0}^{\infty} p \lambda^{p-1} P\left(F^{*}>\lambda\right) d \lambda \leqq \alpha \int_{B} p \lambda^{p-1} P\left(F^{*}>\lambda\right) d \lambda .
$$

(See Lemma 2.6 and the proof of Theorem 3.2 in [2].) Therefore, by (i),

$$
\left\|F^{*}\right\|_{p}^{p} \leqq \alpha \int_{B} p \lambda^{p-1} c P\left(c u^{*}>\lambda\right) d \lambda \leqq c\left\|u^{*}\right\|_{p}^{p} .
$$

This completes the proof.

Remarks. The inequality (3.3) between the distribution functions of $F^{*}$ and $u^{*}$ holds for enough $\lambda$. It need not hold for all $\lambda$ : consider $F(z)=i \log (1-z)$ on $D$ $=\{z:|z|<1\}$ and Brownian motion starting from 0 . Since $u=\operatorname{Re} F$ is bounded, $P\left(c u^{*}>\lambda\right)=0$ for all large $\lambda$. But for any $\lambda, \tau$ and $D_{0}$ may be chosen so that $P\left(F^{*}>\lambda\right)>0$.

Note that M. Riesz's inequalities for the conjugate function follow from Theorem 4. Let $u$ be harmonic in $D=\{z:|z|<1\}$ and $\tilde{u}$ its conjugate satisfying $\tilde{u}(0)=0$. Let $0<r<1$ and $\tau=\inf \left\{t:\left|Z_{t}\right|=r\right\}$ where $\left\{Z_{t}, 0 \leqq t<\infty\right\}$ is Brownian motion starting from 0 . Then, by Theorem 4 ,

$$
\frac{1}{2 \pi} \int_{0}^{2 \pi}\left|\tilde{u}\left(r e^{i \theta}\right)\right|^{p} d \theta=\left\|\tilde{u}\left(Z_{\tau}\right)\right\|_{p}^{p} \leqq c_{p}\left\|_{0 \leqq t<\tau} \mid u\left(Z_{t}\right)\right\|_{p}^{p} .
$$

If $1<p<\infty$, then, by Doob's maximal inequality for martingales,

$$
\left\|\sup _{0 \leqq t<\tau} \mid u\left(Z_{t}\right)\right\|_{p}^{p} \leqq c_{p}\left\|u\left(Z_{\tau}\right)\right\|_{p}^{p}
$$

implying

$$
\int_{0}^{2 \pi}\left|\tilde{u}\left(r e^{i \theta}\right)\right|^{p} d \theta \leqq c_{p} \int_{0}^{2 \pi}\left|u\left(r e^{i \theta}\right)\right|^{p} d \theta
$$

4. Proof of Theorem 2. We make the following remark about the function $\Phi$.

(i) There exists $p>0$ and $C>0$ such that $\lambda^{p} \leqq C \Phi(\lambda)$ for $0 \leqq \lambda \leqq 1$.

The growth condition on $\varphi$ in $(0.3)$ implies $\Phi(2 \lambda) \leqq c \Phi(\lambda)$; therefore $c^{-n} \leqq$ $\Phi\left(2^{-n}\right) / \Phi(1) \leqq C \Phi\left(2^{-(n+1)}\right), n=0,1, \ldots$ If $p=\log _{2} c$, then for $2^{-(n+1)}<\lambda \leqq 2^{-n}$, we have

$$
\lambda^{p} \leqq c^{-n} \leqq C \Phi\left(2^{-(n+1)}\right) \leqq C \Phi(\lambda)
$$

In order to prove Theorem 2 , we may assume that the right-hand side of $(0.4)$ is finite.

(ii) There exists a conjugate harmonic function $\tilde{u}$ such that $\lim _{y \rightarrow \infty} \tilde{u}(x+i y)=0$ uniformly in $x$. 
The fact that the right-hand side of $(0.4)$ is finite implies that $u$ is bounded on every line $\operatorname{Im} z=\varepsilon>0$. Therefore, if $|u(x+i \varepsilon)| \leqq B_{\varepsilon}$, by (i) we have

$$
\begin{aligned}
B_{\varepsilon}^{-p} \int_{-\infty}^{\infty}|u(x+i \varepsilon)|^{p} d x & \leqq C \int_{-\infty}^{\infty} \Phi\left(u / B_{\varepsilon}\right)(x+i \varepsilon) d x \\
& \leqq C_{\varepsilon} \int_{-\infty}^{\infty} \Phi\left(N_{\alpha}(u)\right) d x,
\end{aligned}
$$

so that $u$ is in $L^{p}$ along every $\operatorname{line} \operatorname{Im} z=\varepsilon>0$. Now we may apply the argument given in the proof of Lemma 3 to obtain (ii).

(iii) For any initial point $z_{0}$,

$$
\int \Phi\left(\sup _{0<t<\tau_{0}}\left|\tilde{u}\left(z_{t}\right)-\tilde{u}\left(z_{0}\right)\right|\right) d P_{z_{0}} \leqq C \int \Phi\left(\sup _{0<t<\tau_{0}}\left|u\left(z_{t}\right)-u\left(z_{0}\right)\right|\right) d P_{z_{0}} .
$$

This inequality is analogous to inequality (1.12) and is proved in the same way.

The proof of Theorem 2 may now be completed along the lines of Theorem 1, using inequality (iii) instead of (1.12) and (ii) instead of Lemma 3. We omit further details.

REMARK. Theorem 2 immediately implies the inequality

$$
\sup _{y>0} \int_{-\infty}^{\infty} \Phi(|F(x+i y)|) d x \leqq C \int_{-\infty}^{\infty} \Phi\left(N_{\alpha}(u)\right) d x .
$$

For other related remarks, see $\S 5$.

5. The unit disc. In this section, we state versions of Theorems 1,2 , and 3, for the disc. The proofs are, in general, easier than for the half-plane.

For $0<\sigma<1,0 \leqq \theta \leqq 2 \pi$, let $\Omega_{\sigma}(\theta)$ be the domain bounded by the two tangents from the point $e^{i \theta}$ to the circle $|z|=\sigma$ and by the larger of the two arcs of that circle between the points of contact. If $f(z)$ is defined for $|z|<1$, its nontangential maximal function relative to $\Omega_{\sigma}$ is defined as

$$
N_{\sigma}(f)(\theta)=\sup _{z \in \Omega_{\sigma}(\theta)}|f(z)| .
$$

THEOREM $1^{\prime}$. Let $u$ be harmonic in the unit disc $|z|<1$, $\tilde{u}$ its harmonic conjugate subject to the condition $\tilde{u}(0)=0$, and $F=u+i \tilde{u}$. Then, for all $0<p<\infty$,

$$
c_{\sigma, p} \int_{0}^{2 \pi}\left|N_{\sigma}(u)\right|^{p} d \theta \leqq \sup _{0<r<1} \int_{0}^{2 \pi}\left|F\left(r e^{i \theta}\right)\right|^{p} d \theta \leqq C_{\sigma, p} \int_{0}^{2 \pi}\left|N_{\sigma}(u)\right|^{p} d \theta
$$

THEOREM 2'. Let $\Phi$ be a function as in (0.3). Let $u$ be harmonic in the unit disc and $\tilde{u}$ a conjugate harmonic function with $\tilde{u}(0)=0$. Then, for $0<\sigma<1$,

$$
\int_{0}^{2 \pi} \Phi\left(N_{\sigma}(\tilde{u})\right) d \theta \leqq C \int_{0}^{2 \pi} \Phi\left(N_{\sigma}(u)\right) d \theta
$$

The constant $C$ depends only on $\sigma$ and the growth constant of $\varphi$. 
In the unit disc, the Brownian maximal function of $f$ is defined as

$$
f^{*}=\sup _{0 \leqq t<\tau_{1}}\left|f\left(z_{t}\right)\right|
$$

where $z_{t}$ is complex-valued Brownian motion started at the origin and $\tau_{1}$ is the stopping time $\tau_{1}=\inf \left\{t:\left|z_{t}\right|=1\right\}$.

TheORem 3'. Let $u$ be harmonic in the unit disc $|z|<1$. Then for $0<\sigma<1$ and $\lambda>0$,

$$
c_{\sigma} m\left(N_{\sigma}(u)>\lambda\right) \leqq P\left(u^{*}>\lambda\right) \leqq C_{\sigma} m\left(N_{\sigma}(u)>\lambda\right) .
$$

The proofs of Theorems $1^{\prime}, 2^{\prime}$, and $3^{\prime}$ are, in principle, the same as for the halfplane. A slight exception is the left-hand inequality of Theorem $3^{\prime}$ : the proof of the corresponding inequality for the half-plane involves an argument that makes use of the homogeneity properties of the Poisson kernel for the half-plane (see inequality (1.8)). In the disc, the same argument does not apply. However, we may deduce the left-hand inequality for the disc by conformal mapping from the halfplane. The key to this is an observation, due to Lévy, that two-dimensional Brownian paths are conformally invariant. The argument is as follows:

Let $P^{\theta}$ be the Wiener measure for the process $\left\{z_{t}, t \geqq 0\right\}$ with $z_{0}=0$, conditioned to hit $|z|=1$ for the first time at $z=e^{i \theta}$. If we follow the pattern of proof for the half-plane, the first step is to show an inequality corresponding to (1.8):

$$
P^{\theta}\left(z_{t} \text { does not hit } a_{r}\right) \geqq C_{\sigma}>0,
$$

where $a_{r}$ is the arc formed by the intersection of $\Omega_{\sigma}(\theta)$ with a circle of radius $(1+r) / 2, \sigma<r<1$, inside the unit circle and tangent to it at the point $z=-e^{i \theta}$. It is sufficient to consider the point $z=1$, and to show that

$$
\inf _{\sigma<r<1} P^{0}\left(z_{t} \text { does not hit } a_{r}\right) \geqq C_{\sigma}>0,
$$

where $\left\{a_{r}, \sigma<r<1\right\}$ is the family of arcs corresponding to $z=1$.

Consider the mapping $T(w)=(1+i w)(1-i w)^{-1}$ of the upper half-plane onto the disc; the point $w=i$ is sent to the origin and $w=0$ is sent to $z=1$. A line $\operatorname{Im} z=r^{\prime}$, $0<r^{\prime}<\sigma^{\prime}$, is sent onto a circle of radius $(1+r) / 2, \sigma<r<1$, tangent to the circle $|z|=1$ at the point $z=-1$. Since $T$ is conformal, we may choose $\alpha$ large enough so that $T\left[\Gamma_{\alpha}(0)\right]$ contains the union of the family of $\operatorname{arcs}\left\{a_{r}, \sigma<r<1\right\}$. Let $\left\{w_{t}, t \geqq 0\right\}$ be Brownian motion in the upper half-plane. It follows by the argument leading to inequality (1.8) that

$$
\inf _{0<r^{\prime}<\sigma^{\prime}} P_{i}^{0}\left(w_{t} \text { does not hit } l_{r^{\prime}}\right) \geqq C_{\sigma}>0
$$

where $l_{r}$ is the segment formed by the line $\operatorname{Im} z=r^{\prime}$ across the cone $\Gamma_{\alpha}(0)$. Since, by choice of $\alpha, T\left(l_{r^{\prime}}\right), 0<r^{\prime}<\sigma^{\prime}$, contains the arc $a_{r}, \sigma<r<1$, inequality (5.1) implies

$$
\begin{aligned}
\inf _{\sigma<r<1} P_{i}^{0}\left(T\left(w_{t}\right) \text { does not hit } a_{r}\right) & \geqq \inf _{0<r^{\prime}<\sigma^{\prime}} P_{i}^{0}\left(w_{t} \text { does not hit } l_{r^{\prime}}\right) \\
& \geqq C_{\sigma}>0 .
\end{aligned}
$$


Now we use Lévy's observation that the process $\left\{T\left(w_{t}\right), t \geqq 0\right\}$ has the same distribution as $\left\{z_{t}, t \geqq 0\right\}$ run with a new clock. That is, $\left\{T\left(w_{t}\right), t \geqq 0\right\}$ has the same distribution as $\left\{z_{\tau(t)}, t \geqq 0\right\}$ where $\{\tau(t), t \geqq 0\}$ is some nonnegative increasing process. (See McKean $[9, \S 4.6]$.) Therefore,

$$
\begin{aligned}
\inf _{\sigma<r<1} P_{i}^{0}\left(T\left(w_{t}\right) \text { does not hit } a_{r}\right) & =\inf _{\sigma<r<1} P^{0}\left(z_{t(t)} \text { does not hit } a_{r}\right) \\
& =\inf _{\sigma<r<1} P^{0}\left(z_{t} \text { does not hit } a_{r}\right)
\end{aligned}
$$

since the event in question is clearly invariant under such time changes. From this and (5.2) we obtain

$$
\inf _{\sigma<r<1} P^{0}\left(z_{t} \text { does not hit } a_{r}\right) \geqq C_{\sigma}>0
$$

as desired.

Now we may complete the proof of the left-hand inequality of Theorem $3^{\prime}$ along the lines indicated for the half-plane. More specifically, we show that enough Brownian paths, from the origin, together with their reflections, contain closed curves around points where $|u|>\lambda$. This fact used with the maximum principle allows us to complete the proof by a computation similar to the one given for the half-plane. We omit the details.

Remarks. (a) Theorem $2^{\prime}$ does not hold for the unit disc if the radial maximal function

$$
N_{0}(u)(\theta)=\sup _{0<r<1}\left|u\left(r e^{i \theta}\right)\right|
$$

is used in place of the nontangential maximal function. By Corollary 3 of Bagemihl and Seidel [1], there is a function $F=u+i \tilde{u}$; analytic in the unit disc, such that $\tilde{u}(0)=0$ and, for almost all $\theta, \lim _{r \rightarrow 1} u\left(r e^{i \theta}\right)=0$, but $\lim _{r \rightarrow 1} \tilde{u}\left(r e^{i \theta}\right)=\infty$. In this case, $N_{0}(u)$ is finite almost everywhere and by an elementary construction we may obtain a function $\Phi$ satisfying the growth condition of Theorem 2 , with $\Phi(\infty)=\infty$, so that

$$
\int_{0}^{2 \pi} \Phi\left(N_{0}(\tilde{u})\right) d \theta=\infty
$$

but

$$
\int_{0}^{2 \pi} \Phi\left(N_{0}(u)\right) d \theta \leqq C<\infty
$$

(b) In contrast to inequality (4.1) and part (a) of Theorem 1, it is not possible to prove an inequality of the form

$$
\int_{0}^{2 \pi} \Phi\left(N_{\sigma}(u)\right) d \theta \leqq C \sup _{0<r<1} \int_{0}^{2 \pi} \Phi\left(\left|F\left(r e^{i \theta}\right)\right|\right) d \theta
$$

for function $\Phi$ satisfying the growth conditions of Theorem 2 . This follows from a theorem of Paley and Zygmund [11, Theorem 2]. They show that, given any function $\chi(t)$ that is nonnegative, measurable, bounded on every finite interval of 
$0 \leqq t<\infty$, and satisfies the condition $\chi(t)=o(t)$ as $t$ increases, there exists a function $F(z)$, analytic in $|z|<1$, such that

$$
\sup _{0<r<1} \int_{0}^{2 \pi} \chi\left(\log ^{+}\left|F\left(r e^{i \theta}\right)\right|\right) d \theta \leqq C<\infty,
$$

but the radical limit of $F$ exists only on a set of measure zero.

\section{REFERENCES}

1. F. Bagemihl and W. Seidel, Some boundary properties of analytic functions, Math. Z. 61 (1954), 186-199. MR 16, 460.

2. D. L. Burkholder and R. F. Gundy, Extrapolation and interpolation of quasi-linear operators on martingales, Acta Math. 124 (1970), 249-304.

3. J. L. Doob, Semimartingales and subharmonic functions, Trans. Amer. Math. Soc. 77 (1954), 86-121. MR 16, 269.

4. - Conditional Brownian motion and the boundary limits of harmonic functions, Bull. Soc. Math. France 85 (1957), 431-458. MR 22 \#844.

5. - Boundary limit theorems for a half-space, J. Math. Pures Appl. (9) 37 (1958), 385-392. MR 22 \#845.

6. G. H. Hardy and J. E. Littlewood, A maximal theorem with function theoretic applications, Acta Math. 54 (1930), 81-116.

7. G. A. Hunt, Some theorems concerning Brownian motion, Trans. Amer. Math. Soc. 81 (1956), 294-319. MR 18, 77.

8. - Markoff chains and Martin boundaries, Illinois J. Math. 4 (1960), 313-340. MR 23 \#A691.

9. H. P. McKean, Jr., Stochastic integrals, Probability and Math. Statist., no. 5, Academic Press, New York, 1969. MR 40 \#947.

10. P. W. Millar, Martingale integrals, Trans. Amer. Math. Soc. 133 (1968), 145-166. MR 37 \#2308.

11. R. E. A. C. Paley and A. Zygmund, A note on analytic functions in the unit circle, Proc. Cambridge Philos. Soc. 28 (1932), 266-272.

12. A. Zygmund, Trigonometrical series, 2nd ed., Cambridge Univ. Press, Cambridge, 1959. MR 21 \#6498.

UNIVERSITY OF ILLINOIS, URBANA, IlLINOIS 61801

RUTGERS UNIVERSITY,

New Brunswick, New Jersey 08903 